\title{
CLARKE Y LA FÍSICA DE ROHAULT
}

LAURA BENITEZ

INSTTTUTO DE INVESTIGACIONES FILOSÓFICAS Universidad NaCiONAL AUTÓNंOMa DE MÉxico

\section{Introducción}

Cuando se analizan las nociones de espacio y tiempo en los escritos de Samuel Clarke es indispensable tomar en consideración las notas que, desde la perspectiva newtoniana, le agregó al Sistema de filosofía natural de Jaques Rohault. Tales notas no sólo arrojan luz sobre la controversia newtonianismo versus cartesianismo, sino que muestran las motivaciones e intereses de Clarke en relación con el conocimiento de la naturaleza. Sin embargo, mi afán, en este trabajo, no es meramente descriptivo sino que intento establecer, al menos en parte, que estas teorías antitéticas no representan únicamente dos versiones contrarias del mundo natural sino que, como formas explicativas, revelan compromisos epistemológicos y ontológicos diferentes, que muestran el paso o cambio de vía de reflexión o estilo de pensamiento.

\section{Consideraciones sobre el sistema de filosofía natural de Jacques Rohault}

Si algún libro, particularmente hace converger las tensiones que tuvieron los científicos de la ilustración fue seguramente el Sistema de filosofía natural de Jacques Rohault. ${ }^{1}$

Jacques Rohault fue un físico muy conocido e influyente de la escuela cartesiana, quien acostumbraba exponer los temas de la física de Descartes, acompañándolos de experimentos, cada miércoles en su casa, donde reunía a científicos y otras notables personalidades de la sociedad parisina, entre 1660-1670.

1 Jacques Rohault, A System of Natural Philosophy, ed. facsimilar de la tradución de John y Samuel Clarke, publicada en 1723. Introducción de Larry Laudan, Johnson Reprint Corporation, Nueva York-Londres, 1969, vol. 1, p. ix. 
Es éste un capítulo importante en la historia de la física que, en parte, se refiere a la vigencia del cartesianismo pero, sobre todo, a la introducción del newtonianismo entre 1671 y 1746 . Estas fechas señalan la primera edición en francés del Traité de Rohault y el momento en que el texto dejó, finalmente, de usarse. Hablar sin embargo, de un sólido cartesianismo que hubiese privado hegemónicamente durante la primera mitad del siglo xviI, puede resultar confuso, si no se matiza el papel que, en este "cartesianismo" ilustrado, jugó el texto de Rohault.

Un dato indicativo de la importancia in abstracto del texto es que, entre 1687 y 1740 el libro de Rohault alcanzó 12 reimpresiones, al paso que en el mismo periodo los Principia mathematica (1687) de Newton se remprimieron sólo 4 veces.

Desde la fecha en la que su autor lo diera a la estampa (1671), el libro ya era esperado en Francia por un público nutrido, el de los "Salones", a los que finalmente penetró el cartesianismo, gracias a las dotes didácticas, al amplio conocimiento de la física de su tiempo y a la buena dosis de sentido común de Jacques Rohault. Su fama se extendió rápidamente, primero a Suiza -donde, en 1674, Théophile Bonet lo tradujo al latín-y luego a Inglaterra, tras la introducción de la versión latina en 1682, adoptándose como libro de texto en Cambridge y Oxford en 1692. Esta adopción del cartesianismo tiene que ver con el estado en que se encontraba la ciencia en las universidades inglesas; en efecto, aunque en algunos lugares de Gran Bretaña se conocía bien y se reconocía la superioridad de la física newtoniana, el ambiente general en las universidades era de ortodoxia aristotélica, que se tradujo fácilmente en ortodoxia cartesiana pues, al decir del autor del Traité, en su introducción, su filosofía natural, más que contra Aristóteles, estaba en contra del aristotelismo posterior y mostraba los puntos de franca coincidencia con el "Príncipe de los filósofos".

Hasta aquí podría hablarse de la difusión del cartesianismo por un hombre que, manteniendo la esencial visión del mundo como extensión geométrica, plena de materia sutil, tenía, no obstante, diferencias con el maestro "Cartes" en cuanto a la concepción de la ciencia y la verdad, como veremos más adelante. Sin embargo, el texto no sólo sirvió para difundir el cartesianismo y mostrar las rupturas con la doctrina aristotélica en varios aspectos, sino que sorprendentemente y, de manera gradual, se convirtió igualmente en uno de los textos más importantes para la difusión del newtonianismo. Así, uno podría estimar que, de 1671 a 1692, lo que se difunde es exclusivamente el cartesianismo de Rohault pero, a partir de esa fecha y hasta 1746, el texto alojará también las ideas newtonianas. Esa curiosa transformación se debió a la inquietud de Samuel Clarke de poner al día el Traité, que llevaba más de 20 años publicado. Así se dio, no sólo a la tarea de traducir el Traité 
a un mejor latín, sino de recopilar experimentos nuevos y notas aclaratorias en favor de la física newtoniana. De 1697 a 1713 el Traité se reeditó cuatro veces y experimentó una importante transformación. En efecto, como dice Larry Laudan:

Al principio las notas sólo eran un suplemento experimental del texto y gradualmente se convirtieron en críticas teóricas al Traité desde un punto de vista newtoniano. ${ }^{2}$

El título de la edición inglesa, de 1723, es testigo de esta curiosa transformación: Rohault System of Natural Philosophy. Illustrated with Dr. Samuel Clarke's Notes, Taken Mostly out of Sir Isaac Newton's Philosophy. Como Laudan dice: "El sistema de Rohault no es una filosofía natural sino dos: la de Descartes, interpretada por Rohault y la de Newton, interpretada por Clarke."3

Para resumir esta parte, podemos decir que el Traité tuvo una importante influencia per se desde su impresión hasta los inicios del siglo xviI y los últimos vestigios de ella se pueden todavía rastrear hasta 1730; sin embargo, el texto, como portador de las ideas newtonianas, es objeto de atención desde el inicio del siglo hasta 1746, en que el newtonianismo, consolidado, encuentra otras vías de difusión más propias y adecuadas.

2. Samuel Clarke y el paso de los Principios de la filosofía a los Principios matemáticos de la filosofía natural

Samuel Clarke (1675-1729), se graduó en 1694 en el Caius College de Cambridge, con una tesis en la que defendía la filosofía natural de Newton, en un momento en el que esta universidad era predominantemente cartesiana.

Laudan señala que, a pesar de estar bien dotado para las ciencias naturales y para los clásicos, pues incluso escribió una traducción de la Ilíada, su mayor interés estuvo en la teología; con todo:

Clarke no pudo alejarse de la ciencia [...] personificaba esa curiosa amalgama de piedad y curiosidad científica que caracterizó a muchos de sus compatriotas [yo diría especialmente en la Ilustración Inglesa] pero, a diferencia de muchos de ellos, no era un diletante. ${ }^{4}$

De hecho, algunos trabajos científicos de Clarke se publicaron en las Philosophical Transactions de la Royal Society de Londres.

2 Op. cit., p. x.

3 Ibid., p. xii.

${ }^{4}$ Ibid., p. xxiii. 
En su prefacio del traductor al Traité de Rohault, Clarke explica que este texto ha sido aceptado y ha sido útil para el mundo; sin embargo, sobre el contenido dice que deja al lector el juicio de valor sobre el mismo. Tal vez sea ésta la manera más elegante de decir que, lo allí expuesto, sólo sirve para refutarlo, pues sobre las notas explica ampliamente que:

a) En ellas hay una completa respuesta a las objeciones planteadas al autor (Rohault), sobre lo que parecía carecer de una justa fundamentación. ${ }^{5}$

b) Ha agregado algunos aspectos de filosofía natural, introducidos por filósofos posteriores, naturalmente seleccionando a los mejores escritores.

c) Ha tomado en cuenta algunas observaciones de los filósofos antiguos sobre filosofía natural y también consigna algunas cuestiones de historia natural.

En suma, en esta edición, la más interesante del Traité por sus características, lo menos importante es que Clarke haya enriquecido, con datos de la antigüedad o de su presente, el texto, al paso que es fundamental su crítica a las tesis cartesianas allí vertidas. Tales críticas aparecen como el propósito más importante de la edición de la traducción del texto y no son nada modestas, en la medida en la que pretenden dar una respuesta completa a las objeciones hechas a Rohault. Ésta es la versión suave de la refutación total del cartesianismo, al sustituir la fundamentación, se implica naturalmente, el cambio del cuerpo de la teoría. De otra manera, no se trata de agregados ni de enmiendas, se trata de concepciones teóricas contrarias donde sólo cabe sustituir una por otra.

De manera esquemática, lo que hay que cambiar es la postulación del pleno o identidad materia-extensión, que es el fundamento de la ciencia cartesiana, por la propuesta de la existencia del vacío. Newton percibió muy claramente que toda la teoría cartesiana, la mecánica, la óptica, la astronomía, etc., se sustentaba en la existencia de un mundo pleno de materia y permeado por una sustancia material etérea; así, bastaría con probar la existencia del vacío para echar por tierra la física de Descartes, sólo que, como dice Laudan, "afirmar la existencia del vacío es una cosa y probarla es otra muy diferente". Como el pleno se resistiera a desvanecerse, Newton y sus seguidores buscaron lo que, en palabras de Laudan, llegó a ser el "talón de Aquiles" del cartesianismo, a saber, su teoría de los vórtices. En mi estudio introductorio al Mundo o tratado de la luz, escribí:

5 Ibid., p. 2. 
Muy suscintamente puede decirse que la teoria de los vórtices es la parte central de la cosmología (especulativa) cartesiana, que nos habla del origen, formación y organización del universo. ${ }^{6}$

\section{Y, más adelante:}

En otras palabras, la organización, equilibrio, diversificación del mundo físico, descansa en la teoría de los vórtices pues, una vez que Dios dota de movimiento a la materia (simultáneamente a su creación), el resultado es el cosmos o mundo físico organizado precisamente a partir de los torbellinos que generan, no sólo los diversos cuerpos y los elementos que los constituyen, por acción mecánica, sino el equilibrio entre ellos y, por ende, su ubicación y acción ordenadas. En suma, una vez dotada del movimiento inicial, la materia deviene en cosmos a partir de los remolinos, la teoría resulta enormemente endeble para todo lo que pretende explicar y, por ello, fácilmente criticable. ${ }^{7}$

Según lo consigna Laudan, la demostración de Newton en los libros 2 y 3 de Principia, de que los vórtices no existen, no es concluyente. Esto significó para los newtonianos, tener que modificar el libro 2 ampliamente, durante la segunda mitad del siglo xviI, para encontrar la refutación definitiva a los vórtices, que el autor se había propuesto escribir.

Los argumentos con los que Newton rechaza la teoría de los vórtices son dos de orden astronómico, uno sobre la velocidad de los cometas y el otro sobre la velocidad de los cuerpos celestes, en general, que buscan mostrar la inexistencia del segundo elemento etéreo o fluido, como Newton lo llama, en vista de que no observamos un retraso detectable en el movimiento de los cuerpos, como sería de esperarse, si en todo el universo se hallara tal fluido y uno más, de orden teórico-metodológico, a saber, la imposibilidad de que la teoría de los vórtices sea traducida matemáticamente. Ésta es una cuestión capital, pues Newton dice que no es posible cuantificar la teoría cualitativa de los vórtices de Descartes. Laudan apunta que esta demostración newtoniana:

es muy probablemente el factor de la elección del título de su trabajo: Principios matemáticos de la filosofía natural, una evidente parodia de Los principios de la filosofía. $^{8}$

En lugar del pleno material con sus vórtices, a través de los cuales se explicaba mecánicamente, por choques, el movimiento de los cuerpos, el

6 René Descartes, El mundo o tratado de la luz, trad. Laura Benítez, Instituto de Investigaciones Filosóficas-UNAM, 1986, p. 15.

7 Op. cit., p. 22.

8 Jacques Rohault, op. cit., p. xxvi. 
peso, la luz, etc., tendremos un universo de átomos moviéndose a través del vacio, permeado por fuerzas de atracción y repulsión. La "aparatosa máquina del mundo" se desvanece en los vacíos interestelares, donde fuerzas invisibles e intangibles explicarán ahora el mundo ordenado.

\section{Rohault el cartesiano}

Según algunos de sus biógrafos, las razones que tuvo Rohault para adherirse al cartesianismo no fueron del todo claras; ${ }^{9}$ sin embargo, resultó ser el mejor portavoz de esa doctrina. Ello no significa, empero, que no haya tenido sus propias preocupaciones y preferencias en materia de filosofía natural y de conocimiento en general. Así, en su prefacio al Traité, Rohault expresa que el conocimiento científico es progresivo, algo claramente contrario a las verdades eternas que Descartes propone:

Porque así como un gran número de personas cultivan el mismo arte o ciencia a través de muchas etapas sucesivas, añadiendo su propio ingenio y su propia luz a los descubrimientos antiguos, de quienes vivieron antes que ellos, no es posible sino que tal arte o ciencia reciba gran mejoría y se acerque cada vez más a su más alta perfección. ${ }^{10}$

Si ha habido pocas aportaciones, en los últimos siglos, al campo de la filosofía natural, no es porque su objeto esté más allá de las capacidades humanas, sino porque ha habido errores en el modo de filosofar. En primer lugar, la autoridad concedida a los filósofos de la antigüedad, especialmente a Aristóteles, de quien dice:

Haré únicamente esta observación, que la ilusión, que muchos han tenido de que él [Aristóteles] supo todo lo que podía saberse y que toda ciencia está contenida en sus libros, ha sido la causa de que la mayor parte de los mejores filósofos se apliquen a sí mismos en vano a leer sus trabajos para encontrar en ellos lo que no está y que podrían haber encontrado por su propio ingenio. ${ }^{11}$

El segundo error que señala Rohault es el tratamiento, demasiado metafísico, de las cuestiones de la filosofía natural. Por supuesto, al estudiar cosas muy abstractas y generales, se pierden de vista los efectos particulares. La idea no es acabar con las consideraciones generales, sino no dedicarse únicamente a esas cuestiones. Así, por ejemplo, basta con considerar someramente la naturaleza general del movimiento para pasar, después, a

9 John Schuster, "Rohault, Jacques", en Dictionary of Scientific Biography Charles Scribner's Sons, Nueva York, 1981, vols. 11-12, pp. 506-509.

10 Jacques Rohault, op. cit., p. 3.

11 Ibid., prefacio $s / n$. 
examinar, de manera particular y distinta, todas sus propiedades para poder aplicar éstas al mundo concreto. Al igual que Descartes, desea evitar las cualidades ocultas y las respuestas demasiado generales a los distintos efectos de la naturaleza. Este énfasis en los efectos particulares, si bien puede encontrarse en la sexta parte del Discurso, no es tan marcado como en Rohault, quien reforzará la tesis con su propuesta experimental. La idea general de Rohault es que debe guardarse un equilibrio entre la argumentación y la experimentación, pues si se prescinde de la primera, nos privamos de la libertad de sacar conclusiones y si dejamos a un lado la segunda, nos privamos de la posibilidad de realizar nuevos descubrimientos.

De los experimentos señala tres clases, pero los más útiles para la filosofía natural son aquellos que: "se hacen en consonancia con algún razonamiento, a fin de descubrir si es justo o no". ${ }^{12}$

Un tercer defecto, que Rohault les señala a los filósofos, es no tomar en cuenta las matemáticas, sin las cuales no ejercitamos la mente en la demostración, ni la acostumbramos a discernir lo verdadero de lo falso, lo que se consigue mejor por esta vía, que mediante los preceptos de la lógica.

Sin embargo, la ventaja fundamental de la matemática, según Rohault es que, al enseñarnos a ver las figuras, nos permite comprender sus diferentes propiedades. Esta perspectiva geométrica se extiende a la ontología del mundo natural (macro y micro de manera unificada), así afirma:

$Y$ si las figuras de los cuerpos que percibimos con nuestros sentidos son tan necesarias para los efectos que producen, es razonable pensar que las partes más imperceptibles de la materia, visto que cada una tiene cierta figura, son también capaces de producir ciertos efectos en proporción a su tamaño, como aquellos que vemos que producen los cuerpos más grandes. ${ }^{13}$

En suma, el método que guía a los filósofos modernos en la obtención de importantes resultados en la filosofía natural, es una combinación de razón, experimento y demostraciones matemáticas.

Finalmente, explica que tomó de Aristóteles el rechazo del vacio y de los átomos, pues aunque, por sí misma, la divisibilidad de la materia no parece poder explicar nada, si se considera que las partes no sensibles tienen figura, tamaño y movimiento, por lo cual producen ciertos efectos y que las partes sensibles se componen de aquéllas, al conocer los efectos producidos por las figuras, tamaños, etc., de éstas, se inferirán los de aquéllas.

Rohault finaliza el prefacio de su Sistema de filosofía natural mencionando sus fuentes; así, ha recurrido a los antiguos y a los modernos, pero muy especialmente a Descartes, de quien dice:

12 Ibid.

13 Ibid. 
Pero la persona a quien más he usado en este trabajo y cuyo nombre no he mencionado para nada, para evitar una perpetua repetición, es el famoso "Cartes", cuyo mérito, por el cual deviene más y más conocido para todas las naciones en Europa, como lo ha sido durante mucho tiempo para muchos de los principales estados, hará confesar al mundo entero que Francia es al menos tan feliz, al producir y educar hombres grandes en todas las profesiones, como lo fueron los antiguos griegos. ${ }^{14}$

4. La confrontación de dos explicaciones teóricas: algo más que una cuestión de método

Los partidarios de Newton lo vieron como el representante de una ciencia moderna, progresista y existosa, además de consciente de sus limitaciones y firmemente fundada en el experimento y los datos de la observación experimental. Los cartesianos, aunque admitían la necesidad de desarrollar y mejorar la física cartesiana, consideraron que la "atracción" postulada por Newton constituía un caso de acción a distancia, es decir, se trataba de una cualidad oculta o incluso mágica o milagrosa, no obstante que Newton se defendió muchas veces diciendo que no tomaba "atracción" en un sentido literal y que no le adscribía la gravedad a los cuerpos como su más íntima y esencial propiedad. Además, los cartesianos rechazaron también el espacio vacío, esto es, para ellos, la "nada" a través del que se supone actúa la atracción.

En términos muy generales, el cartesianismo y el newtonianismo difieren en muchos aspectos, tanto téoricos como metodológicos, pero aquí vamos a considerar sólo dos cuestiones, restringidas e importantes, en el manual de Rohault:

1. La concepción del plenismo y el vacuismo.

2. La explicación de los graves.

Como se sabe, Descartes sostiene un plenismo, niega la existencia del vacío y propone que extensión y materia son idénticas. Con ello pretendía eliminar "la nada" y las "fuerzas ocultas" de las explicaciones físicas y cosmológicas.

De este modo, como ya se ha dicho en otra parte, ${ }^{15}$ Descartes era partidario del espacio interno, es decir, consideraba que no había un espacio

14 Ibid.

15 Esto lo he discutido en un artículo en prensa que lleva por título: "Infinitud e ilimitación en René Descartes", asimismo, véase también: "La polémica Descartes-More: ies el espacio interno o externo?" en Memorias del XIII Congreso Interamericano de Filosofía, Universidad de los Andes, Bogotá, Colombia, 1994, pp. 379-384. 
distinto del de los cuerpos, de donde se puede concluir que extensión, materia y espacio son idénticos, puesto que el cuerpo no difiere de la extensión. Por otro lado, como el vacío no es nada, no puede tener determinaciones como la distancia o las dimensiones, que necesitan de un sustancia o sujeto de inherencia. En los Unpublished Scientific Papers, Newton dice que hay que rechazar los argumentos cartesianos, mostrando la diferencia entre cuerpo y extensión pero, sobre todo, es importante mostrar el error que Descartes comete al aplicar a la extensión la vieja división de sustancia y accidente. "[Extentio] habet quendam sibi propium existendi modum qui neque suntantiis, neque accidentibus competit." La extensión, la cual tiene su propio modo de existir, que no coincide ni con la sustancia ni con los accidentes. ${ }^{16}$ Para Newton no es una sustancia, porque no sustenta afecciones y no subsiste absolutamente por sí; en realidad, se trata de cierto efecto de Dios, effectus emanativus, esto es, que aunque no es independiente de Dios, no es propiamente hablando, una criatura producida en su ser por la voluntad de Dios, sino un efecto necesario, pero no un atributo de la divinidad. El espacio es, también, una afección de toda entidad existente, pero no es un accidente. ${ }^{17}$ Por estas dos razones, el espacio no puede ser semejante a la nada.

Para Descartes, el mundo es ilimitado puesto que sólo Dios puede ser considerado como infinito pero, para Newton, el espacio es infinito, ya que no es la propiedad definitoria de la materia, sino una afección en el sentido de condición o suposición ontológica de todo existente. Así, nos dice que:

El espacio se extiende en todas direcciones hasta el infinito, porque no somos capaces de imaginar algún límite en él sin, al mismo tiempo, comprender que hay espacio más allá de él. Y no obstante, todas las líneas, rectas, parabólicas, hiperbólicas y todos los conos y cilindros y todas las otras figuras (que podemos concebir inscritas en él) se extienden hasta el infinito y no están limitadas por ningún lado, aunque deben estar interceptadas aquí y allá por líneas y superficies de todas clases, transversales a ellas. ${ }^{18}$

La primera parte parece de inspiración cartesiana: un espacio al que siempre se le puede añadir algo; sin embargo, se aleja en los ejemplos que sugieren, no un infinito potencial, sino actual. Aún más, Newton dice que no hay por qué considerar el infinito como meramente imaginario, sino como actual, puesto que los puntos en los que se intersectan las figuras geométricas infinitas son reales, aunque se sitúen más allá de los límites del mundo.

16 Newton, Unpublished Scientific Papers, en Alexandre Koyré, Newtonian Studies, The University of Chicago Press, Chicago, 1965, p. 85.

17 Cfr. A. Koyré, op. cit., p. 86, en particular, n. 1.

18 Ibid., p. 87. 
Sin el problema cartesiano de la identificación cuerpo-espacio o materiaextensión, Newton puede, al romperla, asignarles infinitud al espacio y a Dios, que no es lo mismo que asignárselo a la materia y a Dios al mismo tiempo, una consecuencia de la geometrización del mundo físico, que Descartes se cuidó de no admitir. Además, Newton no tenía el problema teológico de identificar la infinitud con la perfección del ser. Koyré dice que, para Newton:

Realmente el espacio no está conectado con la materia sino con el ser, spatium est entis quatenus ens affectio. Ningún ser existe o puede existir que no esté relacionado con el espacio de algún modo. Dios está en todas parte, las mentes creadas están en alguna parte y el cuerpo está en el espacio que ocupa; y lo que quiera que sea que no esté ni en todas partes ni en alguna parte, no existe. ${ }^{19}$

Podemos escuchar, en estas consideraciones, los ecos de la polémica que Descartes sostuvo con Henry More. El nulli ubi para Dios y el alma, que Descartes tiene que sostener consistentemente frente a las burlas de Henry More, quien llamó a los cartesianos nullibistae. El espacio llega a ser, en Newton, afección de los entes igual que el tiempo, de acuerdo con los cuales se determina la cantidad de la existencia de cualquier ser individual, con respecto a la amplitud de su presencia (en cuanto al espacio) y de su perseverancia (en cuanto al tiempo) en el ser. ${ }^{20}$

\section{El problema de la pesantez en la física de Rohault}

Para Descartes la pesantez era una reacción centrípeta de los cuerpos gruesos producida por la presión centrífuga de la materia sutil que tiene lugar en los torbellinos. ${ }^{21}$

En 1669 se suscitó en L'Academie Royal des Sciences, en París, una famosa polémica en la que tomaron parte varios científicos importantes, unos en favor y otros en contra del cartesianismo, Christian Huygens defendió la tesis cartesiana de la pesantez con dos "experimentos", poniendo en vasijas cuerpos de diversa densidad y haciéndoles girar, de tal modo que la materia sutil se ve arrastrada hacia la periferia y la más gruesa es empujada hacia el centro. De sus "experimentos" concluyó que, tal parece como si "un cuerpo menos rápido, en un torbellino más rápido, fuera atraído hacia el centro". No conforme con dar la razón a la explicación cartesiana de la pesantez, Huygens calculó la fuerza centrífuga que un cuerpo desarrolla al

19 Ibid., p. 89.

20 Cfr. A. Koyré, ibid., p. 89.

21 Alfonso Pérez de la Borda, Leibniz y Newton, Universidad Pontificia de Salamanca, Salamanca, 1981, p. 27. 
girar alrededor de otro a cierta distancia: $F=m(4 / T)$. Donde $F=$ fuerza, $M=$ masa y $T=$ tiempo, significando que la Fuerza es igual a cuatro veces la masa sobre el tiempo.

Con esta fórmula intentaba hacer verosímil que la materia sutil desarrolla, en su giro, cierta fuerza centrífuga $X$ pero la materia gruesa, al girar más despacio, tiene una fuerza centrífuga menor a $X$ y así, la fuerza menor es vencida por la mayor, produciendo la reacción centrípeta que exhibe la materia gruesa. De esta forma, la pesantez consiste "en el esfuerzo que la materia fluida hace por alejarse de la tierra en todos los sentidos empujando en su lugar a los cuerpos que no siguen ese movimiento". ${ }^{22}$

Contra estas explicaciones, Gilles Persone de Roberval, ya en 1636, según nos dice Koyré, en una carta que escribió conjuntamente con Étienne Pascal a Fermat, sostenía que:

Es posible que la gravedad sea una cualidad que reside en el mismo cuerpo que cae, puede ser que esté en otro que atrae a aquel que desciende como pasa en la tierra. Puede ser también, y es muy probable que sea una atracción mutua, o un deseo natural de los cuerpos de unirse como es obvio en el caso del hierro y el magneto, que es tal que, si se detiene el magneto, el hierro, si no es impedido, se moverá hacia él. Si el hierro es detenido el magneto se moverá hacia él y si ambos están libres se aproximarán uno a otro recíprocamente. ${ }^{23}$

Roberval también tomó parte en el debate sobre las causas de la pesantez en L'Academie Royal des Sciences de París y, el 7 de agosto de 1669, leyó una memoria en donde vuelve a afirmar que hay tres posibles explicaciones de la gravedad, pero que la más simple es la de la atracción mutua o tendencia de las diferentes partes de la materia a unirse, pero sigue llamando a esta atracción una cualidad oculta. Su gran mérito, sin embargo, es haber escrito su Système du monde sobre la base de la "atracción universal".

Jacques Rohault, por su parte, dedicó el capítulo 28, de su Tratado de física, a la gravedad y la levedad y explica que los nombres de levedad y gravedad han sido usados desde la antigüedad sin comprender realmente en qué consisten semejantes cualidades de los cuerpos que observamos. Así, hay quienes han dicho, siguiendo a Aristóteles, que los cuerpos tienen en sí unos, la tendencia a dirigirse al centro de la tierra (que es además el centro del universo) y otros la tendencia contraria. Sin embargo, hay quienes han considerado que, todos los cuerpos, tienden al centro del universo y, finalmente, hay quienes sostienen que la tendencia de los cuerpos es a alejarse del centro.

23 A. Koyré, op. cit., p. 59. 
A Rohault, la tres opiniones le parecen falsas ya que, siguiendo a Descartes, no admite que haya auténticas tendencias en los propios cuerpos, además de que desconocemos la verdadera causa que los mueve hacia arriba o hacia abajo.

El cartesiano se pregunta entonces en qué consisten la gravedad y la levedad y expresa, como "una de las principales leyes de la naturaleza" que:

las partes de cualquier conjunto que gire en torno a su centro tienen tendencia a alejarse de él. Dicha tendencia es mayor en aquellas partes que tienen mayor movimiento que en las que tienen menos. ${ }^{24}$

Rohault explica que, como en la masa de partes que giran alrededor de su centro, a la que podemos llamar un vórtice, algunas tienen más movimiento que otras - las más pequeñas tienen más movimiento que las más masivas- debe concluirse que las partes con más fuerza o movimiento tienden a alejarse del centro:

pero, las partes que tienen menos fuerza para alejarse, son empujadas con violencia hacia el centro por las que tienen más fuerza; esas partes las llamamos pesadas. ${ }^{25}$

En suma, la gravedad se interpreta como menor levedad pero no como una disposición del cuerpo o partícula al descenso. El peso del cuerpo es proporcional a la cantidad de materia fluida que lo hace descender en su rápido movimiento al alejarse del centro. La gravedad o peso aparece así, como un efecto de la levedad o, si se quiere, la manifestación centrípeta del cuerpo que "cae" es efecto del movimiento centrífugo de la materia sutil.

Por eso, en el párrafo 8, Rohault relata el experimento de Huygens, ya mencionado, de donde concluye que: "la gravedad no es sino menor levedad" y también considera que: "de aquí se sigue que los cuerpos que descienden no tienen ninguna disposición en ellos mismos a descender", lo cual no impide que este movimiento pueda considerarse como natural, puesto que así ha sido establecido por la naturaleza.

Esta curiosa explicación condiciona, también, la propuesta cosmológica cartesiana; en efecto, si consideramos que existen torbellinos de materia sutil que giran con una determinada velocidad, los astros aparecen como cuerpos gruesos o pesados, cuya menor velocidad hace que la materia sutil literalmente los arrastre, de modo que giran en torno a su centro y en torno al sol, no por su propia fuerza, sino por la de la materia sutil que los rodea.

24 Jacques Rohault, op. cit., vol. 2, p. 93.

25 Ibid. 
Con respecto a los astros, Rohault dice que: "la tierra no gira en torno a su centro cada 24 horas por su propia fuerza, sino que es arrastrada por la corriente de una materia fluida que la rodea y que penetra todas sus partes". ${ }^{26}$

Finalmente, en el párrafo 13 Rohault, intenta, siguiendo el mismo modelo mecanicista, la explicación particular de la gravedad de los cuerpos individuales que se reduce, según lo propone, a que un cuerpo que tiene menos materia sutil o fluida tiene menos fuerza para separarse del centro que los cuerpos que tienen más materia sutil.

Frente a estas "explicaciones" de Rohault sobre la gravedad, no se hacen esperar los amplios comentarios de Clarke, quien dice que:

Ésta es una hipótesis muy ingeniosa y muy probable, en la medida en que se piense que el mundo es un pleno, pero ya que se ha mostrado, mediante las muy exactas observaciones de los filósofos modernos, que el mundo no está lleno y que la gravedad es la más antigua y la más universal propiedad de la materia y la más importante para mantener y conservar junta la totalidad del universo, debemos proceder con otro método y proponer otra teoría de la gravedad. ${ }^{27}$

Era fundamental, para esa nueva concepción, dejar claramente establecido que no hay materia sutil en el espacio, ya que la explicación cartesiana de la mínima resistencia de una materia sutil es contraria a la razón y a la experiencia. Si la esencia de la materia no es la extensión, sino la impenetrabilidad, el espacio se libera, dando pie a mejores explicaciones sobre el movimiento de los cuerpos, de los astros, sobre el peso, etcétera.

Clarke, no puede dejar pasar la ocasión para hablar del autor de la nueva propuesta, así dice a continuación:

Para ser breve, el celebrado Sir Isaac Newton, ha llevado a cabo esta investigación con éxito, ya que al suponer la más simple naturaleza de la gravedad, ha establecido el verdadero sistema del mundo más allá de toda controversia y la más clara explicación de los más importantes fenómenos de toda la naturale$\mathrm{za}^{28}$

Clarke resume la opinión de Newton sobre la naturaleza y las propiedades de la gravedad:

1. Cada partícula individual, de todos los cuerpos que sean, gravita hacia cada partícula individual de todos los cuerpos que sean, esto es, se hallan impelidas las unas hacia las otras por la gravedad.

26 Ibid., p. 95.

27 A. Koyré, "Rohault and Clarke on Attraction", en op. cit., p. 170.

28 Jacques Rohault, op. cit., p. 95. 
2. Esta fuerza gravitacional es universal en toda su extensión, esto es, todos los cuerpos cualesquiera, hasta donde sabemos, donde quiera que se encuentren ubicados, no sólo en la tierra sino también en los cielos, ya en la luna o en los planetas, en el sol o en cualquier otro lugar, están dotados de este poder.

3. Esta fuerza es universal en cuanto a las clases de los cuerpos; esto es, la poseen todos los cuerpos cualquiera que sea su figura, forma o textura; ya sean simples o compuestos; fluidos o sólidos; ya sean grandes o pequeños; ya estén en movimiento o en reposo, están dotados con este poder.

4. Esta fuerza es universal también en relación con el tiempo; esto es, siendo todas las otras condiciones las mismas, nunca se incrementa o disminuye.

5. La cantidad de la gravedad, en distancias iguales, es exactamente proporcional a la cantidad de materia en los cuerpos gravitantes.

6. La gravedad, en cuerpos dados, es mayor o menor de acuerdo con la distancia entre estos cuerpos.

7. La proporción del incremento o disminución de la gravedad en los cuerpos que se aproximan o se alejan uno de otro es tal que su fuerza es recíprocamente el doble proporcional al cuadrado de sus distancias. ${ }^{29}$

A partir de esta caracterización de la gravedad, Clarke nos propone cinco conclusiones fundamentales:

A) La gravedad o peso de los cuerpos no es un efecto accidental del movimiento o de alguna materia sutil, como querían los cartesianos, sino una ley originaria y general de toda la materia impresa en ella por Dios, y que se mantiene en ella por un poder eficiente que penetra su sustancia sólida. Clarke hace hincapié en que la gravedad se da en relación con la proporción, precisamente, de la cantidad sólida de los cuerpos. Es, por así decir, el fenómeno propio de la solidez (resistencia o antitipia) que caracteriza a la materia.

B) De esta caracterización de la gravedad, se sigue que hay un verdadero vacío en la naturaleza y, además, que el vacío constituye la mayor parte del mundo natural pues, si supone al mundo como un pleno, se sigue que en él todo cuerpo pesa igual, lo cual es un absurdo.

C) Si se acepta la gravedad se puede explicar el movimiento de los planetas en sus órbitas a partir del primer impulso que Dios les da sin la ayuda de vórtices de materia sutil que sólo retardarían su movimiento. 
D) Clarke recurre a la autoridad de Kepler, quien dijo que: "La gravedad es una afección corporal que es mutua (recíproca) entre los cuerpos de la misma naturaleza." 30

Como se hace evidente, todas estas consecuencias, derivadas de la propuesta de la gravitación universal son, por completo, diferentes y prácticamente opuestas a las tesis cartesianas sostenidas por Rohault.

\section{Conclusión}

De pronto no resulta tan cruel la ironía volteriana. ${ }^{31} \mathrm{Si}$ bien es verdad que Descartes aportó muchos elementos a la física de Newton, no es menos cierto que en este asunto concreto, la propuesta cartesiana parece, al menos, rezagada, no sólo en el contenido sino en el modo de la explicación. El peso excesivo de la teoría cartesiana de la pesantez cae, irremisiblemente ante la dinámica de Newton. Fontenelle había notado que, con Descartes, parecían haberse conjurado las explicaciones mediante "fuerzas ocultas" o atracciones y el vacío, sin embargo, dice, Newton ha regresado a ellas, pero las ha armado de un poder totalmente nuevo. Ello tiene que ver con la forma en la que Descartes concibió que se puede hablar del universo, sin dejar resquicio a cosas no explicadas, plenamente, de manera intelectualmente precisa. Nada debiera escapar a este poder racional. Toda causa debe explicarse y todo principio debe ser claro. Frente a esta forma de entender la explicación del universo, Clarke ha argüido que la identificación de materia con extensión es ininteligible y que el pleno impide explicaciones sencillas de muchos fenómenos, incluso hay experimentos que prueban la inexistencia del pleno. En cuanto a las fuerzas ocultas, la "atracción" no es una causa sino un efecto, pero un efecto universal, del que no se pretenden averiguar las causas pues, en esta forma de hacer ciencia, no interesan principios desconocidos, sino el establecimiento de ciertas regularidades y proporciones de este efecto en los fenómenos naturales. Y, pasando a la ofensiva, los newtonianos podrían decir que los vórtices pueden, fácilmente, llamarse causas ocultas puesto que su existencia nunca se ha demostrado. ${ }^{32}$

30 Cfr. Ibid., n. 1, p. 97.

31 En sus Lettres philosophiques, Voltaire señala lo siguiente:

"Un francés que llega a Londres, encuentra grandes cambios en filosofía como en todo lo demás. Deja el mundo lleno y lo encuentra vacío. En París uno ve el universo compuesto de vórtices de materia sutil; en Londres uno no ve nada de esto. En Paris, es la presión de la luna la que causa la marea; en Inglaterra, es el mar el que gravita hacia la luna. Con los cartesianos, todo se debe a un impulso que nadie entiende; con Newton, es por atracción, cuya causa no es mejor conocida." (Citado por A. Koyré en "Newton and Descartes", en op. cit., p. 55.)

32 Acerca de esto véase A. Koyré, ibid., p. 60. 


\section{Apéndice}

A PROPÓSITO DE SI LA GRAVEDAD ES UNA PROPIEDAD ESENCIAL DE LA MATERIA

A pesar de todo lo que Clarke puntualiza, sobre la importancia de la gravedad en Newton, cabe, según Koyré la pregunta de si la gravedad es una propiedad esencial de la materia para Newton. En efecto, en su Hypotheses Explaining the Profiles of Light, de 1675, Newton sostuvo que no creía que la gravedad fuera "una propiedad innata, esencial e inherente a la materia". Rechazó igualmente la idea de la atracción como acción a distancia. No obstante, Bentley, seguía considerando que: "si una energía constante es infundida por Dios en la materia" como Newton le decía en sus cartas, entonces, "La gravedad debería ser esencial a la materia". ${ }^{33}$

Sin embargo, es importante notar, como lo muestra Koyré, que en Newton no hay una definición única de esta "propiedad" de la materia ya que, en los Principia, en ocasiones se trata de "fuerzas matemáticas", en tanto que en el prefacio del mismo texto dice que estas fuerzas de la naturaleza son "desconocidas" pero están desprovistas de sentido físico y deben entenderse matemáticamente.

Koyré considera que hay cierta irresolución cuando Newton habla de "atracción", "fuerza" o "impulso" para referirse a aquello que sea lo que impulsa a los cuerpos unos contra otros y concluye que, dado lo anterior:

Newton no puede dejar de dar la impresión de estar lidiando, en ambos casos ("impulsos" o "atracciones") con fuerzas físicas semejantes, aun cuando no tome en cuenta o haga abstracción de su realidad física y considere sólo su aspecto matemático. $^{34}$

No obstante sus esfuerzos, los lectores de Newton lo entendieron como sosteniendo la "existencia de fuerzas en los cuerpos". Koyré menciona a Huygens, Leibniz y más extensamente a Locke, quien en su respuesta a la segunda carta del Obispo de Worcester, dice:

La gravitación de la materia hacia la materia, de modos no concebibles por mí, es no sólo una demostración de que Dios, cuando le parece bien, puede poner en los cuerpos poderes y modos de acción que están más allá de lo que puede derivarse de nuestra idea de cuerpo o explicado por lo que conocemos de la materia. ${ }^{35}$

33 Richard Bentley, A Confutation of Atheism from the Origin and Frame of the World, Londres, 1693 (citado por A. Koyré en op. cit., p. 149).

34 Ibid., p. 153.

35 Ibid., p. 155. 
Más aún, los autores del siglo xviI consideraron siempre la gravedad o atracción como una mera cuestión de facto y el problema de su origen fue soslayado. No obstante, parece, que ni la complejidad de las explicaciones newtonianas, ni las perplejidades de los lectores pudieron alterar el "hecho duro" de la gravedad como central a la dinámica newtoniana independientemente del origen de tal fuerza. 